\title{
Cherenkov probes and runaway electrons diagnostics
}

\author{
R. Kwiatkowski ${ }^{a}$, M. Rabinski, M. J. Sadowski, J. Zebrowski, P. Karpinski, \\ COMPASS, TCV
}

National Centre for Nuclear Research, A. Soltana 7, 05-400 Otwock-Swierk, Poland

Received: 14 December 2020 / Accepted: 24 July 2021

(C) The Author(s) 2021

\begin{abstract}
The beams of fast runaway electrons (RE), which are often produced during tokamak discharges, are particularly dangerous and can induce serious damages of the vacuum vessel and internal components of the machine. The proper and fast diagnostics of RE beams is essential for controlling the discharge, e.g., by early mitigation of disruptions and potentially dangerous RE beams. The diagnostics of RE beams is usually based on measurements of the radiation emitted either by these electrons, or as a result of their interactions with plasma and/or vessel walls. Such a radiation is usually recorded by the means of probes placed outside the vacuum vessel. The method developed by our team is based on the probe located inside the vacuum vessel. The probe can be used to detect highly localized RE bunches and to determine their spatial and temporal characteristics. During last few years, the NCBJ team have developed and used the RE diagnostics based on the Cherenkov effect observed in diamond radiators coupled with fast photomultipliers. During the investigated discharges, the probe was inserted into the vacuum vessel, and its head was placed at the plasma edge, where fast RE are expected. A correlation between signals recorded using our probes and other diagnostics, e.g., hard x-ray signals, was also studied. In this paper, we present recent results of the RE measurements by means of Cherenkov probes, which were performed in the COMPASS and TCV tokamaks.
\end{abstract}

\section{Introduction}

Runaway electrons (RE) have been observed in many different tokamaks, i.e., in the large ones (JET, JT-60) and also in medium ones (Tore Supra, COMPASS, TCV) [1-6].

In tokamaks, when an electrical force acting on the electrons due to the toroidal field exceeds the friction between electrons and surrounding plasma, some electrons can escape, change their toroidal trajectories and hit the vacuum vessel walls. Energy of such electrons can reach several or even tens of MeVs.

One can distinguish three main mechanisms of the RE generation: a Dreicer mechanism, an avalanche mechanism, and a hot-tail mechanism. The Dreicer mechanism takes place when Coulomb friction is weaker than the acceleration caused by an induced voltage. The

COMPASS: IPP AS CR, Za Slovankou 3, CZ-18200 Prague, Czechia.

A comprehensive list of TCV consortium members appears in acknowledgement section.

a e-mail: Roch.Kwiatkowski@ncbj.gov.pl (corresponding author) 
avalanche mechanism, meaningful in larger tokamaks, prevails when some fast electron (a so-called runaway seed) collide with slower ones and accelerate them to runaway velocities. The process continues and the number of REs grows exponentially. The hot-tail mechanism takes place when the collisional frequency for sufficiently fast electrons drops rapidly, e.g., during thermal quench, and the electrons are accelerated further by a toroidal field $[1,3,7]$.

High intensity RE bunches could lead to serious damages of in-vessel components. Therefore, investigation of the RE generation is a very important topic for the machine protection. The risk connected with energetic RE beams makes difficult detailed investigation of the considered phenomena. In medium- and small-size tokamaks, the REs are usually generated during the ramp-up or flat-top phase, when plasma electron density is low or a toroidal electric field is higher than the critical field. However, post-disruptive RE beams are not produced when the toroidal magnetic field is lower than some limit (about $2 \mathrm{~T}$ ). In the small- and medium-size tokamaks, such RE beams could be induced by injecting high-Z gas or pellet, even when the toroidal field is below $2 \mathrm{~T}[8,9]$.

The NCBJ team have proposed investigation of RE beams using a Cherenkov effect induced in diamond radiators. The main goal of our measurements was to evaluate the lowenergy part of runaway electron beams, which can be a precursor of a more intense emission. The visible light, recorded with fast photomultiplier, enables the identification of RE bunches and the determination of their spatial and temporal characteristics. The described technique is convenient for direct measurements of localized and very short RE micro-bunches in different tokamaks, what was precisely described for FTU tokamak [10]. It was also shown in the subsection 3.2 in [11] that when the pulsed electron beam from an electron accelerator impacted on diamond radiator of the probe, the Cherenkov effect dominated over the scintillation, cathodoluminescence or X-ray origin signals from radiator due to diamond threshold effect presence. Using a multi-channel probe, where each radiator is covered by a different metallic layer, it is possible to split RE energy spectrum into a few channels with different lower thresholds and roughly to estimate energetic characteristics of the measured RE beams. The total fluence of the recorded RE beam is a low-variable function of the integral of the obtained signals.

\section{Experimental setup}

The developed probe was equipped with three diamond radiators, placed inside the probe head, which was shielded at the sides with a graphite tube. This head was mounted on a movable manipulator, which made it possible to insert the probe into the tokamak vacuum vessel at the chosen position (see Fig. 1). The radiators were covered with metallic layers (filters) of different thickness. Fast electrons penetrating the radiator could emit the Cherenkov radiation, which was detected at the other ends of the optic fibers. An energy threshold of the light generation depended on the diamond optic index and the thickness of the used metallic filter.

The RE beams were measured in two medium-sized tokamaks: in the TCV operated at the EPFL in Lausanne and in the COMPASS operated at the IPP in Prague. The probes, which are described in this paper, were equipped with radiators which could record electrons of energies: above $58 \mathrm{keV}$ (channel \#1), $132 \mathrm{keV}$ (channel \#2), and $197 \mathrm{keV}$ (channel \#3) in the case of COMPASS tokamak, and above $109 \mathrm{keV}$ (channel \#1), $145 \mathrm{keV}$ (channel \#2), and $226 \mathrm{keV}$ (channel \#3) in the case of TCV tokamak. We have prepared two versions of the Cherenkov-type probe, the first—with disk-like diamonds, and the second-with rodlike diamonds. The diamond disks were $8 \mathrm{~mm}$ in diameter and $1.5 \mathrm{~mm}$ in thickness, while 


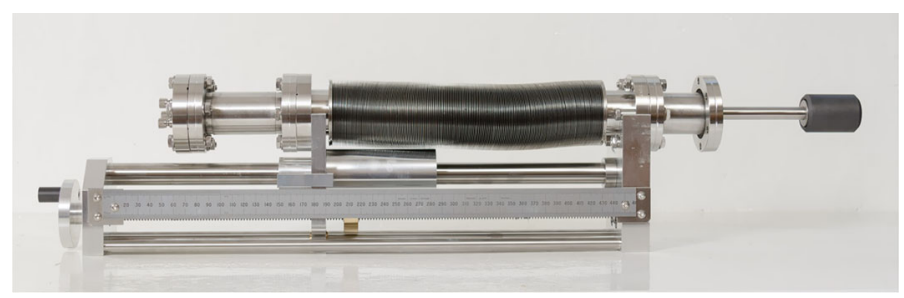

Fig. 1 Vacuum-tight movable manipulator used to mount the Cherenkov probe. This probe could be safely moved within a range of $25 \mathrm{~cm}$
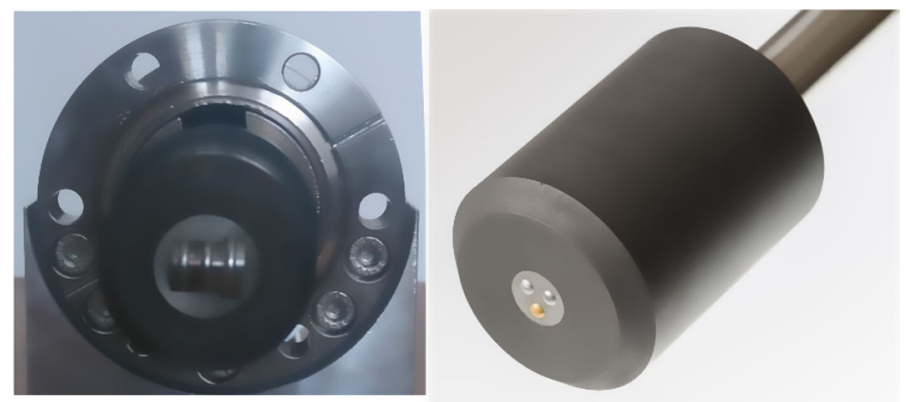

Fig. 2 Front view of the Cherenkov probe head: with the disk-like diamonds (left) and with the rod-like diamonds (right)

the diamond rods have $3 \mathrm{~mm}$ in diameter. The main difference between these two probes was their orientation in relation to the magnetic field lines. The disk-like diamonds should be placed parallel to magnetic lines (electrons travel mostly along these lines), while the rod-like diamonds could be positioned arbitrarily [12-16].

In the both Cherenkov probes, the diamond radiators were coupled with fast photomultipliers by the means of the optical fibers. The signals from PMTs were delivered to an acquisition system of the investigated facility and synchronized with other diagnostics.

The probe used in the COMPASS was equipped with rod-like radiators placed in a triangular pattern in the center of the measuring head, while the probe used in the TCV was equipped with disk-like diamonds which exposed their side-surfaces (see Fig. 2).

\subsection{Probe setup}

The probe used in the COMPASS tokamak was inserted into the vacuum vessel using one of the horizontal ports, and it was connected with three photomultipliers (PTMs) using 20-mlong optical fibers (with a low $\mathrm{OH}$ content). The probe position could be changed in a range from $-5 \mathrm{~cm}$ up to $+20 \mathrm{~cm}$ in relation to the first wall. The deepest position of the probe was set at the position $0 \mathrm{~cm}$, when the diamond radiators were at the limiter edge.

The PMTs were placed inside a special lead bunker, in order to eliminate the X-ray radiation emitted during the investigated discharges. An additional X-ray monitor was placed inside the bunker in order to record the background noise.

The probe used in the TCV was placed in one of the vertical ports of the facility, in the upper part of the toroidal chamber. The probe head could be inserted into the chamber up to a chosen position. Most of the measurements were performed with the probe head positioned 


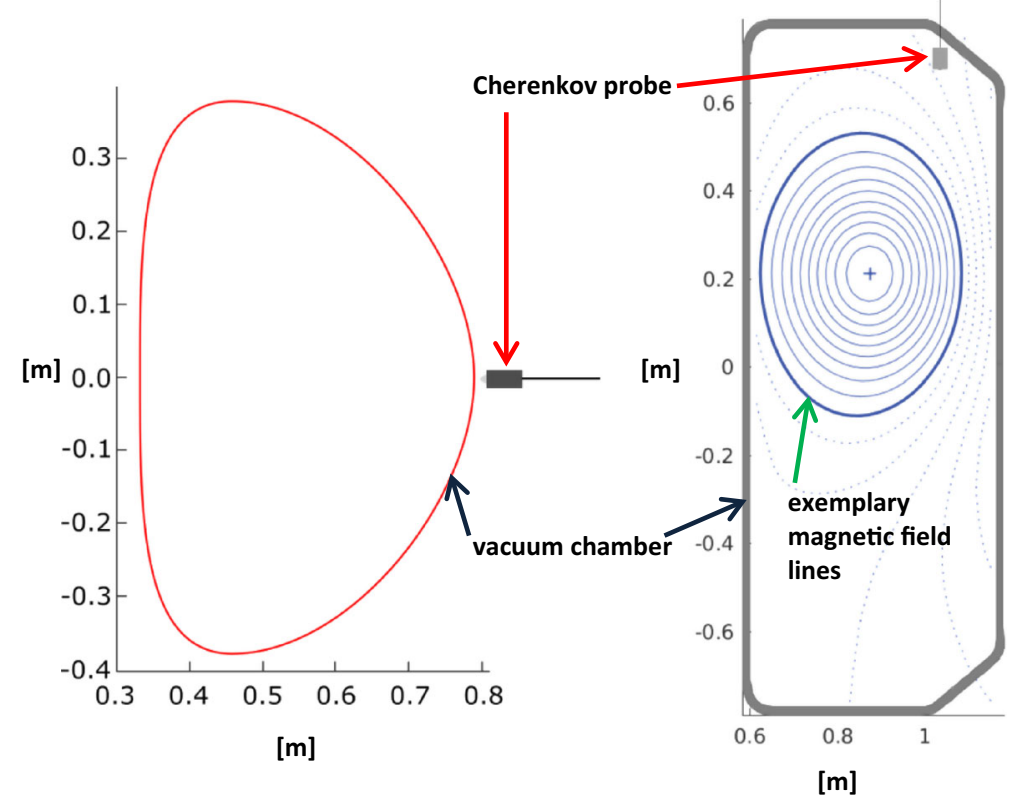

Fig. 3 Scheme of the probe positioning in the vacuum vessels: COMPASS (left) and TCV (right)

at the edge of the vessel wall, when the side-edges of the diamond radiators were just behind the plasma facing components.

The probes were coupled with PMTs using low-OH optic fibers of $40 \mathrm{~m}$ in length. The PMTs, which were placed outside the TCV bunker, did not need any additional shielding.

Schemes showing the probes positioning in the both tokamak experiments are presented in Fig. 3.

\section{Results}

We have recorded signals from the Cherenkov probes during EUROfusion MST1 T08 experimental campaign both at the COMPASS tokamak and TCV facility. The main theme of this campaing was the "Runaway electron beam physics and MGI in support of ITER SPI". In small and medium tokamaks, like the COMPASS and TCV with relatively low currents, the RE beams do not reach high intensity and energy, what makes such devices the suitable facilities to study the generation and controlling of REs. In the reported experiments, the RE beams were initiated by an injection of some high-Z gases: Ar and/or $\mathrm{Kr}$ (during the current ramp-up phase). Comparing the signals recorded by hard X-ray diagnostics and electrical parameters of plasma (i.e., current, density, loop voltage) with the signals obtained from the Cherenkov probe, it was possible to distinguish between signals produced by fast electrons and other sources, like scintillations (in radiators) or signals induced in the used optical fibers or PMTs. It was possible to observe the RE signals from our Cherenkov probes, mainly at the beginning of the discharge and after its disruption.

The RE beams generated during tokamak discharges could also be detected using diagnostics of the hard X-ray generation. The relevant diagnostic system at the COMPASS tokamak 
was called HXR, while that at the TCV_-was noted as PMTX. A comparison of the recorded Cherenkov signals and those obtained from the hard X-ray diagnostics allowed the better identification of the REs signals from the Cherenkov probes to be performed.

Some results obtained from the COMPASS tokamak are shown in Fig. 4. We can observe the high correlation between Cherenkov probe signals and X-rays. Averaged plasma electron density signals during this COMPASS campaign have not good quality and was not taken into consideration. In that case, the RE beams were induced by some Ar-puffing at the beginning of the discharge, and an additional puffing before the current ramp-up, i.e., in shot \#18,915-at $900 \mathrm{~ms}$ and $1100 \mathrm{~ms}$; in shot \#18,824 —at $950 \mathrm{~ms}$ and $1100 \mathrm{~ms}$; and in shot \#18,825—at $950 \mathrm{~ms}$.

Exemplary results obtained from the TCV tokamak are presented in Fig. 5. Similar to the previous case, the observed RE beams were induced by the high- $Z$ gas puffing at different instants: in shot \#61,437-Ne-puffing at $0.47 \mathrm{~s}$ for $2 \mathrm{~ms}$; in shot \#64,603-Kr-puffing at $0.5 \mathrm{~s}$ for $4 \mathrm{~ms}$; in shot \#61,430-Ne-puffing at $1.1 \mathrm{~s}$ for $2 \mathrm{~ms}$; and in shot \#61,432-Ne-puffing at $0.47 \mathrm{~s}$ for $2 \mathrm{~ms}$.

The signals, which were recorded on the first channel only, were produced by REs with energies in the range of (60-130) keV (Fig. 4 for COMPASS). They appeared at the beginning, and in the case of the energy range 110-150 keV for TCV also in the middle of the discharge (see Fig. 5a). The relatively high contribution of REs with energies in the range of (60-130) keV within COMPASS was proved by signal integration value. These values are approximately proportional to electrons fluence recorded by diamond radiator. Similar signals were also observed shortly before the HXR peaks which were generated by high-energy REs. It should be noted that the RE measurements by means of the Cherenkov probes could be used to detect some precursors of the intense RE emission.

Another important feature of the reported Cherenkov measurements, observed particularly at the TCV tokamak, was noticeable differences between signals from different measuring channels. In general, they were inconsistent with the applied filters. It could be an effect of very small and localized electron bunches which could hit one of the diamond radiators only, or various damages and impurity depositions on the radiators surfaces, which might act as additional filters blocking the electrons of energy below a certain threshold.

\section{Discussion}

The Cherenkov probes, which were equipped with small detectors of specific charged particles (electrons), were placed at chosen positions within the investigated tokamaks. It means that we could detect the particles which hit our probes directly. These probes were designed and built in order to make easy to estimate the influence of parasitic neutrons, ions and Xray/gamma radiation signals on the total probes signals. The detection of neutron signal was minimized by application of optical fibers with a low $\mathrm{OH}$ groups content.

The filters upon surfaces of the diamond radiators were used to make possible a rough energy analysis of the recorded electrons. The relative positions of detectors and their sizes, however, made such an analysis possible only in cases when some RE bunches were large enough to cover the three detectors at once. Basing on the signals presented in Fig. 4, one can conclude that during the investigated discharges in the COMPASS tokamak, the RE beams were mainly composed of low-energy electrons $(\mathrm{E}<140 \mathrm{keV})$, and of some energetic ones $(\mathrm{E}>220 \mathrm{keV})$, with a small population of intermediate ones only. 
(a)

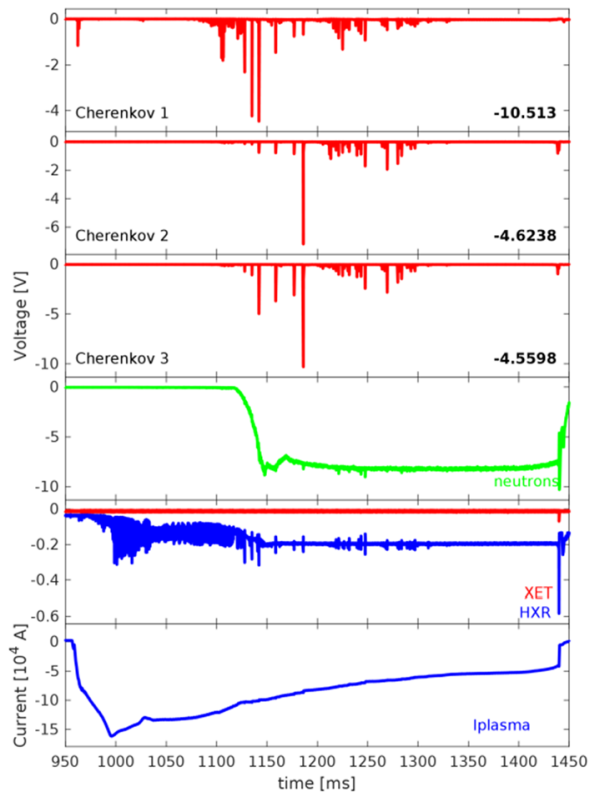

(c)

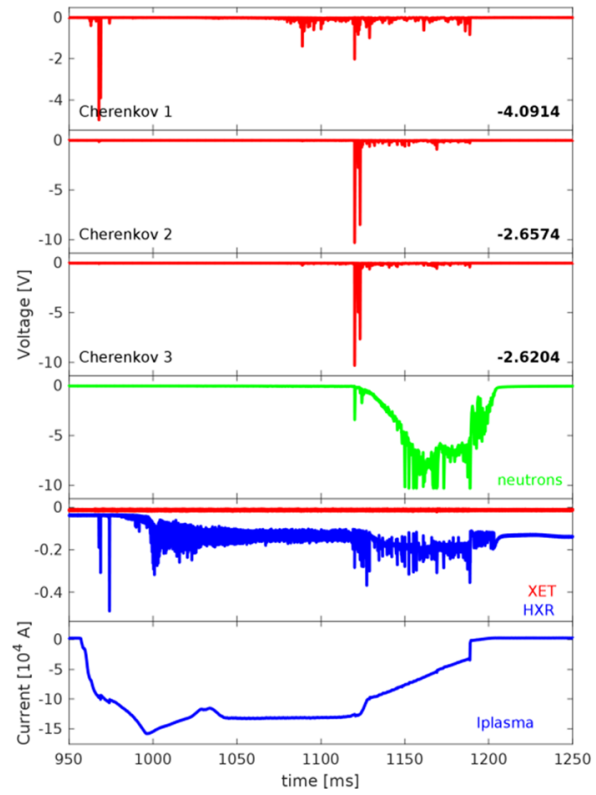

(b) 18825

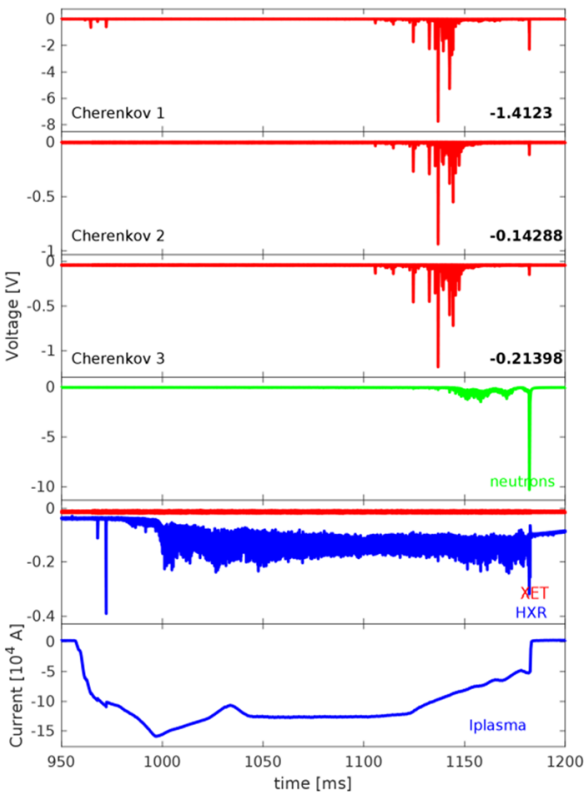

Fig. 4 Signals recorded by means of a Cherenkov probe in the COMPASS tokamak during three different discharges. The values next to Cherenkov signals represent integrals. The lower traces (from middle to bottom) present: neutrons-signals from neutron scintillation probe, XET-signals from the X-ray detector placed close to the PTMs recording Cherenkov signals, HXR - signals from the hard X-ray monitor, $\mathrm{I}_{\text {plasma }}$-the main discharge current 
(a)

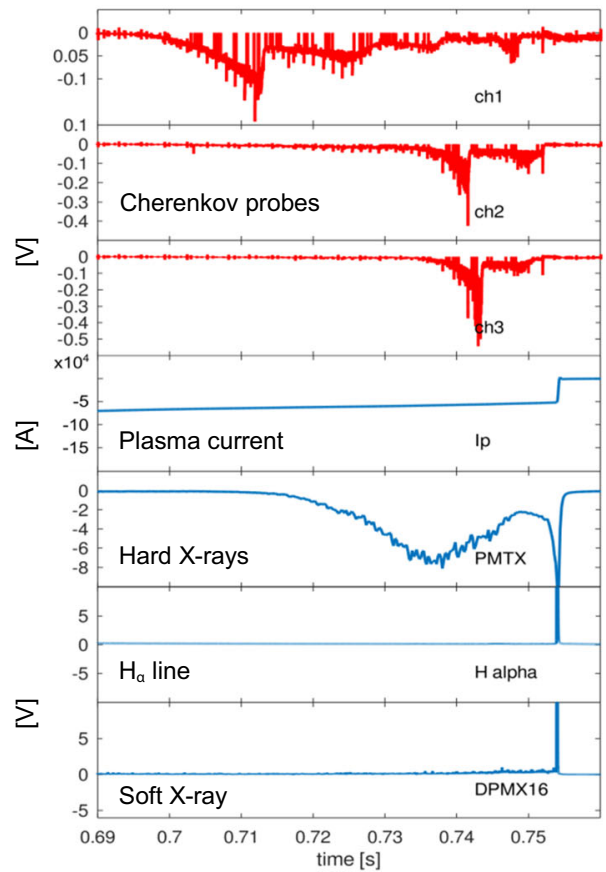

(b)

\#61432

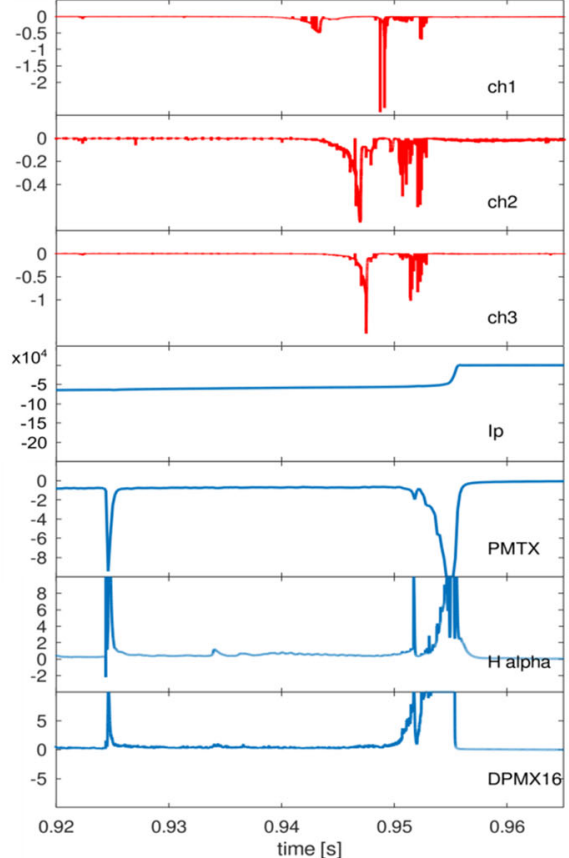

\section{(c)}

\#61437

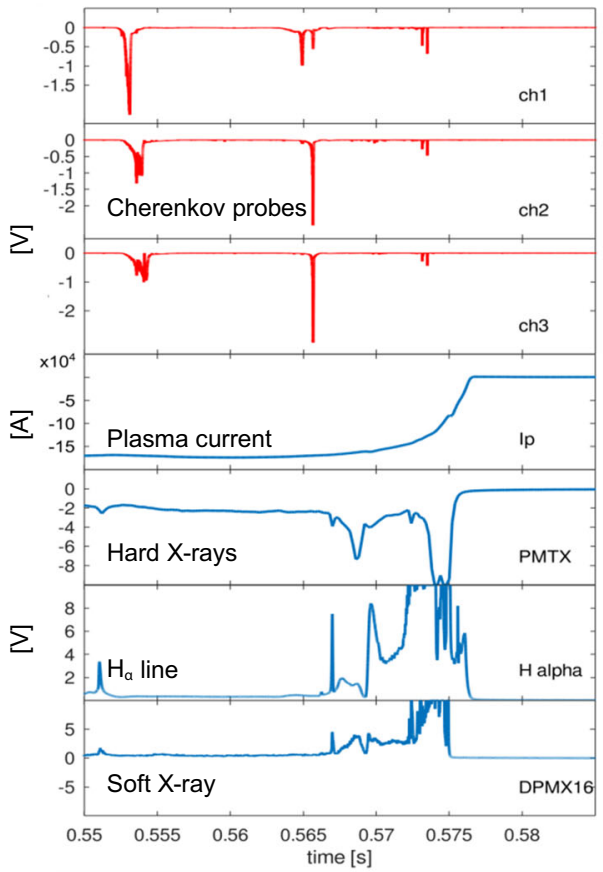

Fig. 5 Signals recorded by means of a Cherenkov probe in the TCV tokamak during three different discharges. The lower traces present the plasma current (Ip), hard X-rays (PMTX), plasma radiation intensity (H alpha), and soft X-rays (DPMX16) 
When the observed RE bunches had smaller dimensions than the detector (radiator) area, or were composed of some micro-bunches with various energies, the detection of separate RE bunches was possible by means of different detectors (see Fig. 5).

The strong X-ray radiation could also be recorded, either by radiators or by optical fibers coupled to PMTs. In such a situation, the recorded signals could have similar intensity in all measuring channels, while the electron bunches hitting the radiators could produce some differences in signals from different channels. In order to estimate and minimize parasite signals, we placed the fast PTMs within a lead bunker equipped with an additional X-ray monitor. We also recorded signals obtained when the optical fibers were disconnected from the diamond radiators. That test showed that a contribution of parasitic signals was low in a comparison with the signals from the diamond radiators.

It can be concluded that the three-channel Cherenkov probe was successfully used to measure the energetic REs emitted during discharges in the TCV and COMPASS tokamaks. The three-channel construction of the Cherenkov-type probe made it possible to observe different behavior of electron bunches of energy ranging (60-140 keV) and those of higher energies, which could be treated as a precursor of more intense RE beams emission.

The observed correlation between the Cherenkov signals and other RE diagnostics showed that the probe was useful for detection of the intense RE beams. However, due to the localized character of the measurements performed, it was possible that there were missed some electron beams, which were recorded with other (e.g., hard X-ray) diagnostics.

The various filters, which were applied in different channels, could be used to perform a rough energy analysis of the investigated REs. The finite sizes and positions of the radiators make such an analysis well-grounded when a single RE bunch hits all of the detectors simultaneously.

In the case, when all the radiators were hit by energetic REs, we could separate low-energy electrons $(\mathrm{E}<140 \mathrm{keV})$ from high-energy ones $(\mathrm{E}>220 \mathrm{keV})$, as shown in Fig. 4.

\section{Conclusions}

The runaway electrons (RE) generated during tokamak discharges could lead to discharge termination and be potentially harmful to the machine. Therefore, the detection and mitigation of REs are of primary importance for tokamak studies.

Our team has developed and used the described probes applicable for the detection of localized RE bunches. Those probes were using the Cherenkov effect induced in the diamond radiators, which could be placed in chosen positions inside the tokamak vacuum vessel.

The developed probes were able to record electrons emitted during plasma disruption, and in some cases also in the middle of the tokamak discharge.

The obtained results are complementary to those obtained with other diagnostics and provide additional information about the localized RE beams.

Acknowledgments This work has been carried out within the framework of the EUROfusion Consortium and has received funding from the Euratom research and training program 2014-2018 and 2019-2020 under Grant Agreement No. 633053. The views and opinions expressed herein do not necessarily reflect those of the European Commission. This scientific work was partly supported by Polish Ministry of Science and Higher Education within the framework of the scientific financial resources in the years 2014-2020 allocated for the realization of the international co-financed project.

TCV collabarator member list: S. Coda: Ecole Polytechnique Fédérale de Lausanne (EPFL), Swiss Plasma Center (SPC), 1015 Lausanne, Switzerland; M. Agostini: Consorzio RFX, Corso Stati Uniti 4, 35127 Padova, Italy; R. Albanese: University of Napoli 'Federico II', Consorzio CREATE, Via Claudio 21, 80125 Napoli, 
Italy; S. Alberti: Ecole Polytechnique Fédérale de Lausanne (EPFL), Swiss Plasma Center (SPC), 1015 Lausanne, Switzerland; E. Alessi: IFP-CNR, via R. Cozzi 53, 20125 Milano, Italy; S. Allan: Department of Physics, Durham University, Durham, DH1 3LE, United Kingdom of Great Britain and Northern Ireland; J. Allcock: Department of Physics, Durham University, Durham, DH1 3LE, United Kingdom of Great Britain and Northern Ireland; R. Ambrosino: University of Napoli Parthenope, Consorzio CREATE, Via Claudio 21, 80125 Napoli, Italy; H. Anand: ITER Organization, Route de Vinon-sur-Verdon, CS 90 046, 13067 St.-Paullez-Durance Cedex, France; Y. Andrèbe: Ecole Polytechnique Fédérale de Lausanne (EPFL), Swiss Plasma Center (SPC), 1015 Lausanne, Switzerland; H. Arnichand: Ecole Polytechnique Fédérale de Lausanne (EPFL), Swiss Plasma Center (SPC), 1015 Lausanne, Switzerland; F. Auriemma: Consorzio RFX, Corso Stati Uniti 4, 35127 Padova, Italy; J.M. Ayllon-Guerola: Centro Nacional de Aceleradores (CNA), Universidad de Sevilla, Junta de Andalucia, Consejo Superior de Investigaciones Cientificas (CSIC), Parque Cientifico y Tecnologico Cartuja, c/Thomas Alva Edison, 7, 41092 Sevilla, Spain, Department of Mechanical Engineering and Manufacturing, University of Seville, Camino de los Descubrimientos s/n, 41092 Sevilla, Spain; F. Bagnato: Ecole Polytechnique Fédérale de Lausanne (EPFL), Swiss Plasma Center (SPC), 1015 Lausanne, Switzerland; J. Ball: Ecole Polytechnique Fédérale de Lausanne (EPFL), Swiss Plasma Center (SPC), 1015 Lausanne, Switzerland; M. Baquero-Ruiz: Ecole Polytechnique Fédérale de Lausanne (EPFL), Swiss Plasma Center (SPC), 1015 Lausanne, Switzerland; A.A. Beletskii: Institute of Plasma Physics, National Science Center, Kharkov Institute of Physics and Technology, 61108 Kharkov, Ukraine ; M. Bernert: Max-Planck-Institut für Plasmaphysik, 85748 Garching, Germany; W. Bin: IFP-CNR, via R. Cozzi 53, 20125 Milano, Italy; P. Blanchard: Ecole Polytechnique Fédérale de Lausanne (EPFL), Swiss Plasma Center (SPC), 1015 Lausanne, Switzerland; T.C. Blanken: Eindhoven University of Technology, PO Box 513, 5600 MB Eindhoven, Netherlands; J.A. Boedo: University of California, San Diego, Energy Research Center, La Jolla, CA 92093, United States of America; O. Bogar: Institute of Plasma Physics AS CR, Za Slovankou 1782/3, 18200 Praha 8, Czech Republic; T. Bolzonella: Consorzio RFX, Corso Stati Uniti 4, 35127 Padova, Italy; F. Bombarda: Unità Tecnica Fusione, ENEA C. R. Frascati, via E. Fermi 45, 00044 Frascati (Roma), Italy; N. Bonanomi: Department of Physics 'G. Occhialini', University of Milano-Bicocca, Piazza Della Scienza 3, 20126 Milano, Italy; F. Bouquey: CEA, IRFM, 13108 Saint Paul Lez Durance, France; C. Bowman: York Plasma Institute, Department of Physics, University of York, Heslington, York, YO10 5DD, United Kingdom of Great Britain and Northern Ireland; D. Brida: MaxPlanck-Institut für Plasmaphysik, 85748 Garching, Germany; J. Bucalossi: CEA, IRFM, 13108 Saint Paul Lez Durance, France; J. Buermans: Laboratory for Plasma Physics, Koninklijke Militaire School—Ecole Royale Militaire, Renaissancelaan 30 Avenue de la Renaissance, 1000 Brussels, Belgium; H. Bufferand: CEA, IRFM, 13108 Saint Paul Lez Durance, France; P. Buratti: Unità Tecnica Fusione, ENEA C. R. Frascati, via E. Fermi 45, 00044 Frascati (Roma), Italy, ; G. Calabró: Department of Economics, Engineering, Society and Business Organization (DEIm), University of Tuscia, Largo dell'Università snc, 01100 Viterbo, Italy; L. Calacci: University of Rome Tor Vergata, via del Politecnico 1, 00133 Rome, Italy; Y. Camenen: Aix-Marseille Université, CNRS, PIIM, 13013 Marseille, France; D. Carnevale: University of Rome Tor Vergata, via del Politecnico 1, 00133 Rome, Italy; F. Carpanese: Ecole Polytechnique Fédérale de Lausanne (EPFL), Swiss Plasma Center (SPC), 1015 Lausanne, Switzerland; M. Carr: CCFE, Culham Science Centre, Abingdon, Oxon, OX14 3DB, United Kingdom of Great Britain and Northern Ireland; L. Carraro: Consorzio RFX, Corso Stati Uniti 4, 35127 Padova, Italy; A. Casolari: Institute of Plasma Physics AS CR, Za Slovankou 1782/3, 18200 Praha 8, Czech Republic; F. Causa: IFP-CNR, via R. Cozzi 53, 20125 Milano, Italy; J. Čeřovský: Institute of Plasma Physics AS CR, Za Slovankou 1782/3, 18200 Praha 8, Czech Republic; O. Chellaï: Ecole Polytechnique Fédérale de Lausanne (EPFL), Swiss Plasma Center (SPC), 1015 Lausanne, Switzerland; P. Chmielewski: Institute of Plasma Physics and Laser Microfusion, Hery 23, 01-497 Warsaw, Poland; D. Choi: Ecole Polytechnique Fédérale de Lausanne (EPFL), Swiss Plasma Center (SPC), 1015 Lausanne, Switzerland; N. Christen: Rudolf Peierls Centre for Theoretical Physics, University of Oxford, Oxford, Oxon, OX1 3PU, United Kingdom of Great Britain and Northern Ireland; G. Ciraolo: CEA, IRFM, 13108 Saint Paul Lez Durance, France; L. Cordaro: Consorzio RFX, Corso Stati Uniti 4, 35127 Padova, Italy; S. Costea: Institut für Ionen- und Angewandte Physik, Universität Innsbruck, Technikerstraße 25, 6020 Innsbruck, Austria; N. Cruz: Instituto de Plasmas e Fusão Nuclear, Instituto Superior Técnico, Universidade de Lisboa, 1049-001 Lisboa, Portugal; A. Czarnecka: Institute of Plasma Physics and Laser Microfusion, Hery 23, 01-497 Warsaw, Poland; A. Dal Molin: Department of Physics 'G. Occhialini', University of Milano-Bicocca, Piazza Della Scienza 3, 20126 Milano, Italy; P. David: Max-Planck-Institut für Plasmaphysik, 85748 Garching, Germany; J. Decker: Ecole Polytechnique Fédérale de Lausanne (EPFL), Swiss Plasma Center (SPC), 1015 Lausanne, Switzerland ; H. De Oliveira: Ecole Polytechnique Fédérale de Lausanne (EPFL), Swiss Plasma Center (SPC), 1015 Lausanne, Switzerland; D. Douai: CEA, IRFM, 13108 Saint Paul Lez Durance, France; M.B. Dreval: Institute of Plasma Physics, National Science Center, Kharkov Institute of Physics and Technology, 61108 Kharkov, Ukraine; B. Dudson: York Plasma Institute, Department of Physics, University of York, Heslington, York, YO10 5DD, United Kingdom of Great Britain and Northern Ireland; M. Dunne: Max-Planck-Institut für Plasmaphysik, 85748 Garching, Germany; B.P. Duval: Ecole Polytechnique Fédérale de Lausanne (EPFL), Swiss Plasma 
Center (SPC), 1015 Lausanne, Switzerland; T. Eich: Max-Planck-Institut für Plasmaphysik, 85748 Garching, Germany; S. Elmore: CCFE, Culham Science Centre, Abingdon, Oxon, OX14 3DB, United Kingdom of Great Britain and Northern Ireland; O. Embréus: Department of Physics, Chalmers University of Technology, 41296 Gothenburg, Sweden; B. Esposito: Unità Tecnica Fusione, ENEA C. R. Frascati, via E. Fermi 45, 00044 Frascati (Roma), Italy; M. Faitsch: Max-Planck-Institut für Plasmaphysik, 85748 Garching, Germany; M. Farník: Institute of Plasma Physics AS CR, Za Slovankou 1782/3, 18200 Praha 8, Czech Republic; A. Fasoli: Ecole Polytechnique Fédérale de Lausanne (EPFL), Swiss Plasma Center (SPC), 1015 Lausanne, Switzerland; N. Fedorczak: CEA, IRFM, 13108 Saint Paul Lez Durance, France; F. Felici: Ecole Polytechnique Fédérale de Lausanne (EPFL), Swiss Plasma Center (SPC), 1015 Lausanne, Switzerland; S. Feng: Department of Physics 'G. Occhialini', University of Milano-Bicocca, Piazza Della Scienza 3, 20126 Milano, Italy; X. Feng: Department of Physics, Durham University, Durham, DH1 3LE, United Kingdom of Great Britain and Northern Ireland; G. Ferró: University of Rome Tor Vergata, via del Politecnico 1, 00133 Rome, Italy; O. Février: Ecole Polytechnique Fédérale de Lausanne (EPFL), Swiss Plasma Center (SPC), 1015 Lausanne, Switzerland; O. Ficker: Institute of Plasma Physics AS CR, Za Slovankou 1782/3, 18200 Praha 8, Czech Republic; A. Fil: York Plasma Institute, Department of Physics, University of York, Heslington, York, YO10 5DD, United Kingdom of Great Britain and Northern Ireland; M. Fontana: Ecole Polytechnique Fédérale de Lausanne (EPFL), Swiss Plasma Center (SPC), 1015 Lausanne, Switzerland; L. Frassinetti: Fusion Plasma Physics, EES, KTH, 10044 Stockholm, Sweden; I. Furno: 3Ecole Polytechnique Fédérale de Lausanne (EPFL), Swiss Plasma Center (SPC), 1015 Lausanne, Switzerland; D.S. Gahle: Department of Physics SUPA, University of Strathclyde, Glasgow, G4 0NG, United Kingdom of Great Britain and Northern Ireland; D. Galassi: Ecole Polytechnique Fédérale de Lausanne (EPFL), Swiss Plasma Center (SPC), 1015 Lausanne, Switzerland; K. Gałązka: Institute of Plasma Physics and Laser Microfusion, Hery 23, 01-497 Warsaw, Poland; A. Gallo: CEA, IRFM, 13108 Saint Paul Lez Durance, France; C. Galperti: Ecole Polytechnique Fédérale de Lausanne (EPFL), Swiss Plasma Center (SPC), 1015 Lausanne, Switzerland; S. Garavaglia: IFP-CNR, via R. Cozzi 53, 20125 Milano, Italy; J. Garcia: CEA, IRFM, 13108 Saint Paul Lez Durance, France; M. Garcia-Muñoz: 10Centro Nacional de Aceleradores (CNA), Universidad de Sevilla, Junta de Andalucia, Consejo Superior de Investigaciones Cientificas (CSIC), Parque Cientifico y Tecnologico Cartuja, c/Thomas Alva Edison, 7 , 41092 Sevilla, Spain, Department of Atomic, Molecular and Nuclear Physics, University of Seville, 41012 Sevilla, Spain; A.J. Garrido: Faculty of Engineering, University of the Basque Country (UPV/EHU), Paseo Rafael Moreno 3, 48013 Bilbao, Spain; I. Garrido: Faculty of Engineering, University of the Basque Country (UPV/EHU), Paseo Rafael Moreno 3, 48013 Bilbao, Spain; J. Gath: Department of Physics, Technical University of Denmark, Bldg 309, 2800 Kgs Lyngby, Denmark; B. Geiger: Max-Planck-Institut für Plasmaphysik, Teilinstitut Greifswald, 17491 Greifswald, Germany; G. Giruzzi: CEA, IRFM, 13108 Saint Paul Lez Durance, France; M. Gobbin: Consorzio RFX, Corso Stati Uniti 4, 35127 Padova, Italy; T.P. Goodman: Ecole Polytechnique Fédérale de Lausanne (EPFL), Swiss Plasma Center (SPC), 1015 Lausanne, Switzerland; G. Gorini: Department of Physics 'G. Occhialini', University of Milano-Bicocca, Piazza Della Scienza 3, 20126 Milano, Italy; M. Gospodarczyk: Department of Economics, Engineering, Society and Business Organization (DEIm), University of Tuscia, Largo dell'Università snc, 01100 Viterbo, Italy; G. Granucci: IFP-CNR, via R. Cozzi 53, 20125 Milano, Italy; J.P. Graves: Ecole Polytechnique Fédérale de Lausanne (EPFL), Swiss Plasma Center (SPC), 1015 Lausanne, Switzerland; M. Gruca: Institute of Plasma Physics and Laser Microfusion, Hery 23, 01-497 Warsaw, Poland; T. Gyergyek: Jožef Stefan Institute, Jamova 39, 1000 Ljubljana, Slovenia; A. Hakola: VTT Technical Research Centre of Finland Ltd, PO Box 1000, 02044 VTT, Finland; T. Happel: Max-PlanckInstitut für Plasmaphysik, 85748 Garching, Germany; G.F. Harrer: Institute of Applied Physics, T.U. Wien, Fusion@ÖAW, Wiedner Hauptstr. 8-10, 1040 Vienna, Austria; J. Harrison: CCFE, Culham Science Centre, Abingdon, Oxon, OX14 3DB, United Kingdom of Great Britain and Northern Ireland; E. Havlíčková: CCFE, Culham Science Centre, Abingdon, Oxon, OX14 3DB, United Kingdom of Great Britain and Northern Ireland; J. Hawke: Ecole Polytechnique Fédérale de Lausanne (EPFL), Swiss Plasma Center (SPC), 1015 Lausanne, Switzerland; S. Henderson: CCFE, Culham Science Centre, Abingdon, Oxon, OX14 3DB, United Kingdom of Great Britain and Northern Ireland; P. Hennequin: Laboratoire de Physique des Plasmas, CNRS UMR7648, Ecole Polytechnique, 91128 Palaiseau, France; L. Hesslow: Department of Physics, Chalmers University of Technology, 41296 Gothenburg, Sweden; D. Hogeweij: 41FOM Institute DIFFER 'Dutch Institute for Fundamental Energy Research', 5600 HH Eindhoven, Netherlands; J.-Ph. Hogge: Ecole Polytechnique Fédérale de Lausanne (EPFL), Swiss Plasma Center (SPC), 1015 Lausanne, Switzerland; C. Hopf: Max-Planck-Institut für Plasmaphysik, 85748 Garching, Germany; M. Hoppe: Department of Physics, Chalmers University of Technology, 41296 Gothenburg, Sweden; J. Horáček: Institute of Plasma Physics AS CR, Za Slovankou 1782/3, 18200 Praha 8, Czech Republic; Z. Huang: Max-Planck-Institut für Plasmaphysik, Teilinstitut Greifswald, 17491 Greifswald, Germany; A. Hubbard: Plasma Science and Fusion Center, Massachusetts Institute of Technology, Cambridge, MA 02139, United States of America; A. Iantchenko: Ecole Polytechnique Fédérale de Lausanne (EPFL), Swiss Plasma Center (SPC), 1015 Lausanne, Switzerland; V. Igochine: 13, ; P. Innocente: 4, ; C. Ionita Schrittwieser: Institut für Ionen- und Angewandte Physik, Universität Innsbruck, Technikerstraße 
25, 6020 Innsbruck, Austria; H. Isliker: Aristotle University of Thessaloniki, 54124 Thessaloniki, Greece; R. Jacquier: Ecole Polytechnique Fédérale de Lausanne (EPFL), Swiss Plasma Center (SPC), 1015 Lausanne, Switzerland; A. Jardin: Institute of Nuclear Physics Polish Academy of Sciences (IFJ PAN), 31-342 Krakow, Poland; A. Kappatou: Max-Planck-Institut für Plasmaphysik, 85748 Garching, Germany; A. Karpushov: Ecole Polytechnique Fédérale de Lausanne (EPFL), Swiss Plasma Center (SPC), 1015 Lausanne, Switzerland; P.-V. Kazantzidis: National Technical University of Athens, 10682 Athens, Greece; D. Keeling: CCFE, Culham Science Centre, Abingdon, Oxon, OX14 3DB, United Kingdom of Great Britain and Northern Ireland; N. Kirneva: Institute of Physics of Tokamaks, National Research Center 'Kurchatov Institute', 123182 Kurchatov Sq., 1, Moscow, Russian Federation, National Research Nuclear University MEPhI (Moscow Engineering Physics Institute), 115409, Kashirskoe Sh., 31, Moscow, Russian Federation; M. Komm: Institute of Plasma Physics AS CR, Za Slovankou 1782/3, 18200 Praha 8, Czech Republic; M. Kong: Ecole Polytechnique Fédérale de Lausanne (EPFL), Swiss Plasma Center (SPC), 1015 Lausanne, Switzerland; J. Kovacic: Jožef Stefan Institute, Jamova 39, 1000 Ljubljana, Slovenia; N. Krawczyk: Institute of Plasma Physics and Laser Microfusion, Hery 23, 01-497 Warsaw, Poland; O. Kudlacek: Max-Planck-Institut für Plasmaphysik, 85748 Garching, Germany; T. Kurki-Suonio: Department of Applied Physics, Aalto University, 00076 Aalto, Finland; R. Kwiatkowski: National Centre for Nuclear Research (NCBJ), 05-400 Otwock, Poland; B. Labit: Ecole Polytechnique Fédérale de Lausanne (EPFL), Swiss Plasma Center (SPC), 1015 Lausanne, Switzerland; E. Lazzaro: IFP-CNR, via R. Cozzi 53, 20125 Milano, Italy; B. Linehan: Plasma Science and Fusion Center, Massachusetts Institute of Technology, Cambridge, MA 02139, United States of America; B. Lipschultz: York Plasma Institute, Department of Physics, University of York, Heslington, York, YO10 5DD, United Kingdom of Great Britain and Northern Ireland; X. Llobet: Ecole Polytechnique Fédérale de Lausanne (EPFL), Swiss Plasma Center (SPC), 1015 Lausanne, Switzerland; R. Lombroni: Department of Economics, Engineering, Society and Business Organization (DEIm), University of Tuscia, Largo dell'Università snc, 01100 Viterbo, Italy; V.P. Loschiavo: University of Napoli 'Federico II', Consorzio CREATE, Via Claudio 21, 80125 Napoli, Italy; T. Lunt: Max-Planck-Institut für Plasmaphysik, 85748 Garching, Germany; E. Macusova: Institute of Plasma Physics AS CR, Za Slovankou 1782/3, 18200 Praha 8, Czech Republic; J. Madsen: Department of Physics, Technical University of Denmark, Bldg 309, 2800 Kgs Lyngby, Denmark; E. Maljaars: Eindhoven University of Technology, PO Box 513, 5600 MB Eindhoven, Netherlands; P. Mantica: IFP-CNR, via R. Cozzi 53, 20125 Milano, Italy; M. Maraschek: Max-Planck-Institut für Plasmaphysik, 85748 Garching, Germany; C. Marchetto: IFP-CNR, via R. Cozzi 53, 20125 Milano, Italy; A. Marco: Advanced Design \& Analysis Department, IDOM, 48015 Bilbao, Spain; A. Mariani: IFP-CNR, via R. Cozzi 53, 20125 Milano, Italy; C. Marini: General Atomics, PO Box 85608, San Diego, CA 92186, United States of America; Y. Martin: Ecole Polytechnique Fédérale de Lausanne (EPFL), Swiss Plasma Center (SPC), 1015 Lausanne, Switzerland; F. Matos: Max-Planck-Institut für Plasmaphysik, 85748 Garching, Germany; R. Maurizio: Ecole Polytechnique Fédérale de Lausanne (EPFL), Swiss Plasma Center (SPC), 1015 Lausanne, Switzerland; B. Mavkov: Department of Electrical and Electronic Engineering, University of Melbourne, Victoria 3010, Australia; D. Mazon: CEA, IRFM, 13108 Saint Paul Lez Durance, France; P. McCarthy: Department of Physics, University College Cork, Cork, Ireland; R. McDermott: Max-Planck-Institut für Plasmaphysik, 85748 Garching, Germany; V. Menkovski: Eindhoven University of Technology, PO Box 513, 5600 MB Eindhoven, Netherlands; A. Merle: Ecole Polytechnique Fédérale de Lausanne (EPFL), Swiss Plasma Center (SPC), 1015 Lausanne, Switzerland; H. Meyer: CCFE, Culham Science Centre, Abingdon, Oxon, OX14 3DB, United Kingdom of Great Britain and Northern Ireland; D. Micheletti: IFP-CNR, via R. Cozzi 53, 20125 Milano, Italy; F. Militello: CCFE, Culham Science Centre, Abingdon, Oxon, OX14 3DB, United Kingdom of Great Britain and Northern Ireland; K. Mitosinkova: Institute of Plasma Physics AS CR, Za Slovankou 1782/3, 18200 Praha 8, Czech Republic; J. Mlynář: Institute of Plasma Physics AS CR, Za Slovankou 1782/3, 18200 Praha 8, Czech Republic; V. Moiseenko: Institute of Plasma Physics, National Science Center, Kharkov Institute of Physics and Technology, 61108 Kharkov, Ukraine; P.A. Molina Cabrera: Ecole Polytechnique Fédérale de Lausanne (EPFL), Swiss Plasma Center (SPC), 1015 Lausanne, Switzerland; J. Morales: CEA, IRFM, 13108 Saint Paul Lez Durance, France; J.-M. Moret: Ecole Polytechnique Fédérale de Lausanne (EPFL), Swiss Plasma Center (SPC), 1015 Lausanne, Switzerland; A. Moro: IFP-CNR, via R. Cozzi 53, 20125 Milano, Italy; R.T. Mumgaard: Plasma Science and Fusion Center, Massachusetts Institute of Technology, Cambridge, MA 02139, United States of America; V. Naulin: Department of Physics, Technical University of Denmark, Bldg 309, 2800 Kgs Lyngby, Denmark; R.D. Nem: Department of Physics, Technical University of Denmark, Bldg 309, 2800 Kgs Lyngby, Denmark; F. Nespoli: Aix-Marseille Université, CNRS, PIIM, 13013 Marseille, France; A.H. Nielsen: Department of Physics, Technical University of Denmark, Bldg 309, 2800 Kgs Lyngby, Denmark; S.K. Nielsen: Department of Physics, Technical University of Denmark, Bldg 309, 2800 Kgs Lyngby, Denmark; M. Nocente: Department of Physics 'G. Occhialini', University of Milano-Bicocca, Piazza Della Scienza 3, 20126 Milano, Italy; S. Nowak: IFP-CNR, via R. Cozzi 53, 20125 Milano, Italy; N. Offeddu: Ecole Polytechnique Fédérale de Lausanne (EPFL), Swiss Plasma Center (SPC), 1015 Lausanne, Switzerland; F.P. Orsitto: University of Napoli 'Federico II', Consorzio CREATE, Via Claudio 21, 80125 Napoli, Italy; R. Paccagnella: Consorzio 
RFX, Corso Stati Uniti 4, 35127 Padova, Italy; A. Palha: Eindhoven University of Technology, PO Box 513, 5600 MB Eindhoven, Netherlands; G. Papp: Max-Planck-Institut für Plasmaphysik, 85748 Garching, Germany; A. Pau: Department of Electrical and Electronic Engineering, University of Cagliari, Piazza d'Armi, 09123 Cagliari, Italy; R.O. Pavlichenko: Institute of Plasma Physics, National Science Center, Kharkov Institute of Physics and Technology, 61108 Kharkov, Ukraine; A. Perek: FOM Institute DIFFER'Dutch Institute for Fundamental Energy Research', 5600 HH Eindhoven, Netherlands; V. Pericoli Ridolfini: Institute of Plasma Physics and Laser Microfusion, Hery 23, 01-497 Warsaw, Poland; F. Pesamosca: Ecole Polytechnique Fédérale de Lausanne (EPFL), Swiss Plasma Center (SPC), 1015 Lausanne, Switzerland; V. Piergotti: Unità Tecnica Fusione, ENEA C. R. Frascati, via E. Fermi 45, 00044 Frascati (Roma), Italy; L. Pigatto: Consorzio RFX, Corso Stati Uniti 4, 35127 Padova, Italy; P. Piovesan: Consorzio RFX, Corso Stati Uniti 4, 35127 Padova, Italy; C. Piron: Consorzio RFX, Corso Stati Uniti 4, 35127 Padova, Italy; V. Plyusnin: Instituto de Plasmas e Fusão Nuclear, Instituto Superior Técnico, Universidade de Lisboa, 1049-001 Lisboa, Portugal; E. Poli: Max-Planck-Institut für Plasmaphysik, 85748 Garching, Germany; L. Porte: Ecole Polytechnique Fédérale de Lausanne (EPFL), Swiss Plasma Center (SPC), 1015 Lausanne, Switzerland; G. Pucella: Unità Tecnica Fusione, ENEA C. R. Frascati, via E. Fermi 45, 00044 Frascati (Roma), Italy; M.E. Puiatti: Consorzio RFX, Corso Stati Uniti 4, 35127 Padova, Italy; T. Pütterich: Max-Planck-Institut für Plasmaphysik, 85748 Garching, Germany; M. Rabinski: National Centre for Nuclear Research (NCBJ), 05-400 Otwock, Poland; J. Juul Rasmussen: Department of Physics, Technical University of Denmark, Bldg 309, 2800 Kgs Lyngby, Denmark; T. Ravensbergen: 14Eindhoven University of Technology, PO Box 513, 5600 MB Eindhoven, Netherlands, FOM Institute DIFFER 'Dutch Institute for Fundamental Energy Research', 5600 HH Eindhoven, Netherlands, ; M. Reich: Max-Planck-Institut für Plasmaphysik, 85748 Garching, Germany; H. Reimerdes: Ecole Polytechnique Fédérale de Lausanne (EPFL), Swiss Plasma Center (SPC), 1015 Lausanne, Switzerland; F. Reimold: Forschungszentrum Jülich GmbH, Institut für Energie- und Klimaforschung —Plasmaphysik, 52425 Jülich, Germany; C. Reux: CEA, IRFM, 13108 Saint Paul Lez Durance, France; D. Ricci: University of Napoli 'Federico II', Consorzio CREATE, Via Claudio 21, 80125 Napoli, Italy P. Ricci: Ecole Polytechnique Fédérale de Lausanne (EPFL), Swiss Plasma Center (SPC), 1015 Lausanne, Switzerland; N. Rispoli: IFP-CNR, via R. Cozzi 53, 20125 Milano, Italy; J. Rosato: Aix-Marseille Université, CNRS, PIIM, 13013 Marseille, France; S. Saarelma: CCFE, Culham Science Centre, Abingdon, Oxon, OX14 3DB, United Kingdom of Great Britain and Northern Ireland; M. Salewski: Department of Physics, Technical University of Denmark, Bldg 309, 2800 Kgs Lyngby, Denmark; A. Salmi: VTT Technical Research Centre of Finland Ltd, PO Box 1000, 02044 VTT, Finland; O. Sauter: Ecole Polytechnique Fédérale de Lausanne (EPFL), Swiss Plasma Center (SPC), 1015 Lausanne, Switzerland; M. Scheffer: Eindhoven University of Technology, PO Box 513, 5600 MB Eindhoven, Netherlands; Ch. Schlatter: Ecole Polytechnique Fédérale de Lausanne (EPFL), Swiss Plasma Center (SPC), 1015 Lausanne, Switzerland; B.S. Schneider: Institut für Ionen- und Angewandte Physik, Universität Innsbruck, Technikerstraße 25, 6020 Innsbruck, Austria; R. Schrittwieser: Institut für Ionen- und Angewandte Physik, Universität Innsbruck, Technikerstraße 25, 6020 Innsbruck, Austria; S. Sharapov: CCFE, Culham Science Centre, Abingdon, Oxon, OX14 3DB, United Kingdom of Great Britain and Northern Ireland; R.R. Sheeba: Aix-Marseille Université, CNRS, PIIM, 13013 Marseille, France; U. Sheikh: Ecole Polytechnique Fédérale de Lausanne (EPFL), Swiss Plasma Center (SPC), 1015 Lausanne, Switzerland; R. Shousha: Eindhoven University of Technology, PO Box 513, 5600 MB Eindhoven, Netherlands; M. Silva: Ecole Polytechnique Fédérale de Lausanne (EPFL), Swiss Plasma Center (SPC), 1015 Lausanne, Switzerland; J. Sinha: ITER Organization, Route de Vinon-sur-Verdon, CS 90 046, 13067 St.-Paul-lez-Durance Cedex, France; C. Sozzi: IFP-CNR, via R. Cozzi 53, 20125 Milano, Italy; M. Spolaore: Consorzio RFX, Corso Stati Uniti 4, 35127 Padova, Italy; L. Stipani: Ecole Polytechnique Fédérale de Lausanne (EPFL), Swiss Plasma Center (SPC), 1015 Lausanne, Switzerland; P. Strand: Department of Physics, Chalmers University of Technology, 41296 Gothenburg, Sweden; T. Tala: VTT Technical Research Centre of Finland Ltd, PO Box 1000, 02044 VTT, Finland; A.S. Tema Biwole: Ecole Polytechnique Fédérale de Lausanne (EPFL), Swiss Plasma Center (SPC), 1015 Lausanne, Switzerland; A.A. Teplukhina: Princeton University, Princeton, NJ 08544, United States of America; D. Testa: Ecole Polytechnique Fédérale de Lausanne (EPFL), Swiss Plasma Center (SPC), 1015 Lausanne, Switzerland; C. Theiler: Ecole Polytechnique Fédérale de Lausanne (EPFL), Swiss Plasma Center (SPC), 1015 Lausanne, Switzerland; A. Thornton: CCFE, Culham Science Centre, Abingdon, Oxon, OX14 3DB, United Kingdom of Great Britain and Northern Ireland; G. Tomaž: Jožef Stefan Institute, Jamova 39, 1000 Ljubljana, Slovenia; M. Tomes: Institute of Plasma Physics AS CR, Za Slovankou 1782/3, 18200 Praha 8, Czech Republic; M.Q. Tran: Ecole Polytechnique Fédérale de Lausanne (EPFL), Swiss Plasma Center (SPC), 1015 Lausanne, Switzerland; C. Tsironis: National Technical University of Athens, 10682 Athens, Greece; C.K. Tsui: Ecole Polytechnique Fédérale de Lausanne (EPFL), Swiss Plasma Center (SPC), 1015 Lausanne, Switzerland, University of California, San Diego, Energy Research Center, La Jolla, CA 92093, United States of America; J. Urban: Institute of Plasma Physics AS CR, Za Slovankou 1782/3, 18200 Praha 8, Czech Republic; M. Valisa: Consorzio RFX, Corso Stati Uniti 4, 35127 Padova, Italy; M. Vallar: Consorzio RFX, Corso Stati Uniti 4, 35127 Padova, Italy; D. Van Vugt: Eindhoven University of Technology, 
PO Box 513, 5600 MB Eindhoven, Netherlands; S. Vartanian: CEA, IRFM, 13108 Saint Paul Lez Durance, France; O. Vasilovici: Institut für Ionen- und Angewandte Physik, Universität Innsbruck, Technikerstraße 25, 6020 Innsbruck, Austria; K. Verhaegh: York Plasma Institute, Department of Physics, University of York, Heslington, York, YO10 5DD, United Kingdom of Great Britain and Northern Ireland; L. Vermare: Laboratoire de Physique des Plasmas, CNRS UMR7648, Ecole Polytechnique, 91128 Palaiseau, France; N. Vianello: Consorzio RFX, Corso Stati Uniti 4, 35127 Padova, Italy; E. Viezzer: 10Centro Nacional de Aceleradores (CNA), Universidad de Sevilla, Junta de Andalucia, Consejo Superior de Investigaciones Cientificas (CSIC), Parque Cientifico y Tecnologico Cartuja, c/Thomas Alva Edison, 7, 41092 Sevilla, Spain, Department of Atomic, Molecular and Nuclear Physics, University of Seville, 41012 Sevilla, Spain; W.A.J. Vijvers: FOM Institute DIFFER 'Dutch Institute for Fundamental Energy Research', 5600 HH Eindhoven, Netherlands; F. Villone: University of Napoli 'Federico II', Consorzio CREATE, Via Claudio 21, 80125 Napoli, Italy; I. Voitsekhovitch: CCFE, Culham Science Centre, Abingdon, Oxon, OX14 3DB, United Kingdom of Great Britain and Northern Ireland; N.M.T. Vu: Ecole Polytechnique Fédérale de Lausanne (EPFL), Swiss Plasma Center (SPC), 1015 Lausanne, Switzerland; N. Walkden: CCFE, Culham Science Centre, Abingdon, Oxon, OX14 3DB, United Kingdom of Great Britain and Northern Ireland; T. Wauters: Laboratory for Plasma Physics, Koninklijke Militaire School-Ecole Royale Militaire, Renaissancelaan 30 Avenue de la Renaissance, 1000 Brussels, Belgium; M. Weiland: Max-Planck-Institut für Plasmaphysik, 85748 Garching, Germany; H. Weisen: Ecole Polytechnique Fédérale de Lausanne (EPFL), Swiss Plasma Center (SPC), 1015 Lausanne, Switzerland; M. Wensing: Ecole Polytechnique Fédérale de Lausanne (EPFL), Swiss Plasma Center (SPC), 1015 Lausanne, Switzerland; M. Wiesenberger: Department of Physics, Technical University of Denmark, Bldg 309, 2800 Kgs Lyngby, Denmark; G. Wilkie: Department of Physics, Chalmers University of Technology, 41296 Gothenburg, Sweden; M. Wischmeier: Max-Planck-Institut für Plasmaphysik, 85748 Garching, Germany; K. Wu: Department of Economics, Engineering, Society and Business Organization (DEIm), University of Tuscia, Largo dell'Università snc, 01100 Viterbo, Italy; M. Yoshida: National Institutes for Quantum and Radiological Science and Technology, Naka, Ibaraki 311-0193, Japan; R. Zagorski: Institute of Plasma Physics and Laser Microfusion, Hery 23, 01-497 Warsaw, Poland; P. Zanca: Consorzio RFX, Corso Stati Uniti 4, 35127 Padova, Italy; J. Zebrowski: National Centre for Nuclear Research (NCBJ), 05-400 Otwock, Poland; A. Zisis: Department of Physics, National and Kapodistrian University of Athens, 15784 Athens, Greece; M. Zuin: Consorzio RFX, Corso Stati Uniti 4, 35127 Padova, Italy; EUROfusion MST1 Team: Department of Applied Physics, Aalto University, 00076 Aalto, Finland.

\section{Declarations}

Conflict of interest The authors declare that they have no known competing financial interests or personal relationships that could have appeared to influence the work reported in this paper.

Open Access This article is licensed under a Creative Commons Attribution 4.0 International License, which permits use, sharing, adaptation, distribution and reproduction in any medium or format, as long as you give appropriate credit to the original author(s) and the source, provide a link to the Creative Commons licence, and indicate if changes were made. The images or other third party material in this article are included in the article's Creative Commons licence, unless indicated otherwise in a credit line to the material. If material is not included in the article's Creative Commons licence and your intended use is not permitted by statutory regulation or exceeds the permitted use, you will need to obtain permission directly from the copyright holder. To view a copy of this licence, visit http://creativecommons.org/licenses/by/4.0/.

\section{References}

1. R. J. E. Jaspers (1995) Relativistic runaway electrons in tokamak plasmas, PhD Thesis, Technical University, Eindhoven

2. B. Esposito et al., Dynamics of high energy runaway electrons in the Frascati Tokamak Upgrade. Phys. Plasmas 10(6), 2350-2423 (2003)

3. J. Wesson, Tokamaks (Oxford University Press, New York, 2004)

4. O. Ficker, Runaway electrons on the COMPASS and GOLEM tokamaks, CVUT Research task, (2014) https://physics.fjfi.cvut.cz/publications/FTTF/VU_Ondrej_Ficker.pdf

5. H. Knoepfel, D.A. Spong, Runaway electrons in toroidal discharges. Nucl. Fus. 19(6), 785 (1979) 
6. V.V. Plyusnin et al., Study of runaway electron generation during major disruptions in JET. Nucl. Fus. 46(2), 277 (2006)

7. H. Dreicer, Electron and ion runaway in a fully ionized gas, I. Phys. Rev. 115, 238-249 (1959)

8. R.D. Gill et al., Behaviour of disruption generated runaways in JET. Nucl. Fus. 42(8), 1039-1044 (2002)

9. M. Vlainic et al., Post-disruptive runaway electron beams in the COMPASS tokamak. J. Plasma Phys. 81, 475810506 (2015)

10. F. Causa et al., Cherenkov emission provides detailed picture of non-thermal electron dynamics in the presence of magnetic islands. Nucl. Fus. 55, 123021 (2015)

11. F. Bagnato et al., Triple Cherenkov probe measurements on FTU: calibration and runaway energy spectra. Plasma Phys. Control. Fusion 60, 115010 (2018)

12. J. Zebrowski et al., Studies of runaway electrons via Cherenkov effect in tokamaks. J. Phys. Conf. Ser. 959, 012002 (2018)

13. M. Rabinski et al., Development of a Cherenkov-type diagnostic system to study runaway electrons within the COMPASS tokamak. JINST 12, C10014 (2017)

14. V.V. Plyusnin et al., Use of Cherenkov-type detectors for measurements of runaway electrons in the ISTTOK tokamak. Rev. Sci. Instrum. 79, 10F505 (2008)

15. M.J. Sadowski et al., Adaptation of selected diagnostic techniques to magnetic confinement fusion experiments. Czech. J. Phys. 54, C74-C80 (2004)

16. L. Jakubowski et al., Study of electron beams within ISTTOK tokamak by means of a multi-channel Cherenkov detector; their correlation with hard X-rays. Nucl. Instrum. Meth. A 623, 686 (2010) 